\title{
Incidence and Causes of Tibial Component Loosening Based on Level of Obesity in a Large Consecutive Cohort of Cemented Primary TKAs without Stem Extensions
}

\author{
Lilly Longawa, ${ }^{1}$ Leonard T. Buller, ${ }^{2,3}$ Mary Ziemba-Davis, ${ }^{2}$ R. Michael Meneghini ${ }^{2,3}$
}

${ }^{1}$ Indiana University School of Medicine

${ }^{2}$ Indiana University Health Physicians, Orthopaedics

${ }^{3}$ Indiana University School of Medicine, Department of Orthopaedic Surgery

Background and Hypothesis: Aseptic loosening is one of the most common failure mechanisms of total knee arthroplasty (TKA) requiring revision. The influence of obesity on tibial component aseptic loosening remains unknown. Some surgeons advocate the addition of a tibial stem extension to reduce the risk of loosening. The purpose of this study was to quantify the incidence and causes of revision for tibial component loosening in a large consecutive cohort of cemented primary TKAs without stem extensions based upon level of obesity.

Experimental Design or Project Methods: 534 consecutive cemented primary TKAs performed between 2016 and 2018 by one surgeon were retrospectively reviewed. Procedures were performed using consistent surgical, perioperative medical, and pain-control protocols. All tibial implants were tapered thin keeled designs without stem extensions and implanted with low viscosity cement. Medical records were examined and all-cause revisions of the index surgery were documented.

Results: After exclusions for confounds, 525 TKAs were analyzed. Mean age and BMI were 67.8 years and $33.9 \mathrm{~kg} / \mathrm{m}^{2}$ respectively and $72 \%$ were female. Mean follow-up was 19.1 (SD 10.5) months and $48 \%$ had minimum two-year follow-up. $41.3 \%$ of patients had a BMI greater than $35 \mathrm{~kg} / \mathrm{m}^{2}$ and $21.9 \%$ above $40 \mathrm{~kg} / \mathrm{m}^{2}$. There were 11 revisions in 10 patients, $1.9 \%$ out of all TKAs performed. Five TKAs required both-component revision and three involved the femoral component only. No tibial component revisions were performed for aseptic loosening.

Conclusion and Potential Impact: None of the standard tibial implants with a keeled design without stem extensions failed in a patient population with greater than $40 \%$ obesity. Despite some suggesting tibial stem extensions should be used in obese TKA patients, these findings suggest that tibial component failure in obese patients may be design specific and routinely utilizing stem extensions may not be warranted and could result in deleterious bone loss at revision if required for reasons other than aseptic loosening. 